\title{
The Information Behaviour of Social Workers: Needs, Seeking, Acquiring and Using Information in Practice
}

\author{
Niamh Flanagan (1) *
}

Department of Applied Social Studies, Laraghbryan House, Maynooth University, Maynooth, Ireland

*Correspondence to Niamh Flanagan, Department of Applied Social Studies, Laraghbryan House, Maynooth University, Maynooth, Co. Kildare W23, F2H6, Ireland. E-mail niamh.flanagan@mu.ie

\begin{abstract}
In the debate about what informs social work practice, research remains the dominant discourse. However, the relationship between research and social work practice has always been an uneasy one, arguably passed from other clinical disciplines without resizing to fit social work. Even as social work research matures as a discipline it represents one element in a much broader composite which informs practice. This article takes a unique step back from the traditional research-practice discourse and examines the broader information landscape of social work practice, asking how practitioners inform their practice, rather than how research informs practice. This study explores the information needs that prompt practitioners to search for information, the strategies they employ, their acquisition of information and the uses to which the information is put. This study aims to elucidate the information behaviour with a view to improving dissemination and use. Findings demonstrate that the social work information base is substantially broader than has been suggested. Practitioners employ a pragmatic palette of strategies to navigate the breadth of information that supports practice, from research through to knowledge sharing. This article proposes that a pragmatic framework of information behaviour is required to accurately reflect the information behaviour of social workers.
\end{abstract}

Keywords: Information behaviour, information seeking, information-base, knowledgebase, social work

Accepted: October 2019 


\title{
Introduction
}

In order for the service-users to be empowered, practitioners need to be empowered and that power is derived from the information. (GannonLeary, 2006)

The issue of 'practice information' lies at the crux of key debates in social work: professionalisation, the knowledge base, research-to-practice and the evidence-based practice debate. Yet little attention has been paid to how practice information is sought, acquired or used, or indeed, in what circumstances a need for information is identified or dismissed. Social work literature has generally focused on whether social workers read and/or research (Gannon-Leary, 2006; Horder, 2006). All too often the final judgement is a quantification of information seeking, which is of limited explanatory value.

Drawing on the information science field of 'information behaviour' (IB) (Wilson, 1997), this study uniquely proposes a broader exploration of information in social work. Moving beyond reading and research to develop a model of the pragmatic information practices which social workers employ to support their work in the fast-paced world of frontline work, this study explores how social workers seek information, why they seek, what they seek and how they use the acquired information.

\section{Background}

\begin{abstract}
By opening the mind to alternative framings of knowledge and its use emerging in the knowledge utilisation field, it becomes possible to explore the link between research and practice in ways more suitable to decision making in social work. In this way, meaningful enhancements to research use in social work may be achieved. (Heinsch et al., 2016, p. 103)
\end{abstract}

Discourse on what informs social work practice has predominantly focused on the dichotomy of 'experiential knowledge' and 'research' with Trevithick (2008, p. 1217) adding the third domain 'theoretical', which can derive inductively from knowledge or deductively from research. While the substantial scholarship on critical reflection evidences its centrality in social work (Fook et al., 2015), the emphasis on research-based information had traditionally cast social work in a less favourable light and the research-to-practice gap remains a well-documented concern (Sharland, 2012). Despite the perceived importance of research for social work, literature suggests that: undertaking empirical research is not a central task for social workers (Gray et al., 2015); a reading culture is 
not in evidence (Buckley and Whelan, 2009; Iversen and Heggen, 2016); practitioners do not make significant use of research to underpin practice (Buckley and Whelan, 2009; Avby et al., 2017); and do not value it in the way health professionals do (Booth et al., 2003, p. 192). Indeed, social work research has been characterized as 'young' or 'emergent' (Sharland, 2012, p. 217). Notwithstanding these deficit-based characterisations, research remains the gold standard of social work information, and there would appear to be growing evidence of an emerging enthusiasm for social work research (Buckley et al., 2014; Heinsch and Gray, 2016). This does, however, underline the importance of addressing the wider information-to-practice landscape to understand social work information practices from an asset-based rather than deficit-based perspective.

Exploration of the factors underpinning the usage of research and wider information in social work points to six practical and cultural influences acting against the development of a research and reading culture in social work: first, the issue of insufficient time (Beddoe, 2011; Gray et al., 2015); second, primacy of experiential knowledge over research knowledge (Walter et al., 2004; Gannon-Leary, 2006); third, opposing views about whether responsibility for promoting and using research lies with organisations (Booth et al., 2003) or if practitioners need a greater sense of personal accountability for managing their own continued learning (Randall, 2002); fourth, responses to the researchpractice gap have largely tended to place the burden of utilisation on practitioners by increasing production and training. Rosen (2003) posits that this only contributes to further and unnecessary alienation between researchers and practitioners. Instead, Tozer and Ray (1999) advocate that research should be managed, presented and disseminated in such a way that practitioners are empowered by research findings, rather than alienated from them. More recently, calls have reiterated the need for a shift in focus from production of research toward a better understanding of the ways in which practitioners access and use 'information' (GannonLeary, 2006; Heinsch et al., 2016; Kelly, 2017).

Moving beyond the focus on research-based evidence and taking a broader perspective on what informs social work, a fifth aspect is evident. A perceived lack of access to relevant information (Buckley and Whelan, 2009; Holden et al., 2012) and limited access to quality technology (Kelly, 2017) have resulted in social workers being described as 'information poor' (Gannon-Leary, 2006, p. 122). Poor access is compounded by alleged skills deficits in information seeking (GannonLeary, 2006). While practical barriers to information have been afforded scholarly attention, there has been less focus on the inter-related cultural aspects of social work that may act against the flow of information and evidence. A final relevant feature of the profession is its primarily oral or interpersonal mode of practice (Horder, 2006; Tsang, 2007). It has 
been reported that information seeking in social work practice is largely verbal, via face-to-face contact (Gannon-Leary, 2006; Buckley and Whelan, 2009), and knowledge dissemination is largely oral through supervision and training (Harrison et al., 2004). This preference for 'socially relationally-derived knowledge' should be capitalised on rather than surmounted (Greenhalgh and Wieringa, 2011; Heinsch et al., 2016).

Evolving approaches, such as 'Translational and Implementational Science', which address the interface between research and practice, increasingly advocate for more evidence of the needs and characteristics of the target audience (Fixen et al., 2005). These disciplines argue that a better understanding of how practitioners seek and use information to support their practice is necessary (Walter et al., 2004). This understanding can inform a more effective system of information flow which will support evidence-informed decision making. It is posited that information and research dissemination strategies can be developed which work in tandem with social workers' practices rather than mirroring the practices of other professions such as medicine, psychiatry or psychology.

\section{Information behaviour}

This study's approach was influenced by two distinct fields of study, IB and 'Applied Behaviour Analysis' (ABA). The principles of ABA involve micro-level understanding of the functionality of behaviour through: studying real-time behaviour; analysing the steps involved in a behaviour; and then modifying these steps where appropriate (Kearney, 2008). As an ancillary function of social work, seeking and using information must be considered within the context of its functionality, i.e. the role information plays in the broader tasks of social work. ABA establishes functionality by examining the context of the event, examining what happens before (antecedents) and after the behaviour (consequences) to understand what triggers and reinforces the behaviour. Adopting these ABA principles, this study explored the needs (antecedents), seeking (behaviour), acquisition and use (consequences) of information in social work.

This study also drew on macro-level theoretical models from human IB in an attempt to frame social workers' activities. IB is defined in terms of a number of domains including 'how people need, seek, manage, give and use information in different contexts' (Fisher, 2005, p. xix). Of the various domains of IB, 'seeking' has been afforded the most attention. It is typically conceptualised as a process, a form of problem solving involving stages (e.g. Kuhlthau, 1991; Ellis, 1993). This process approach offers the potential to divide social workers' information-seeking practices into steps that can be analysed individually as per the ABA approach. These theories typically include such stages as defining 
a question, browsing and chaining through literature, differentiating between sources and verifying facts, before winding up the search. While social workers may employ these classic search strategies in some aspects of their work, it seems counterintuitive that these models could encapsulate the breadth of social work IB.

Although less developed than 'information seeking', distinct bodies of scholarship on all four dimensions of IB-need, seeking, acquisition and use-continue to evolve (Case and Given, 2016). Models, such as that of Wilson (1997), offer a macro-perspective, outlining the overarching process of IB, from identification of a need, through seeking, information use or failure, any reiteration of the search process and contextual factors. While Wilson's widely accepted model provides a comprehensive overview of IB, its principal limitation is its need for 'specification by analytical concepts' (Ingwersen and Järvelin, 2005, p. 67). As such, although it describes the general process, it cannot describe the range of strategies that social workers employ to address their various information needs.

While existing models do not capture the nature of social work practices, there are, at best, only a handful of, somewhat dated, studies of the IB of social workers (Wilson and Streatfield, 1977). This clear gap in theoretical and empirical consideration of how social work practitioners use information to support their practice seems an anathema in the current information age.

\section{Methodology}

To address the overarching question of what information practices social workers employ, this study addressed the following questions:

1. What 'needs' prompt social workers to seek information?

2. What 'search' strategies do social workers employ?

3. How do social workers 'acquire' information?

4. How do social workers 'use' the information they gather?

This study used a mixed-method design employing audio-diaries in phase I and Critical Incident Technique (CIT) interviews in phase II to gather qualitative data.

\section{Research sample}

A purposive sample of sixteen research-interested social workers participated in the study. Suitable practitioners, identified by reputation, were invited to participate. Given the commitment required by the study, forty-six invitations were required to achieve a sample of sixteen 
Table 1 Profile of study sample and National Social Work Workforce

\begin{tabular}{llc}
\hline & $\begin{array}{l}\text { Study sample } \\
(n=16), \%\end{array}$ & $\begin{array}{l}\text { National Social Work } \\
\text { Workforce }(N=2488)^{\mathrm{a}}, \%\end{array}$ \\
\hline Female & 75 & 83 \\
$<35$ years of age & 50 & 42 \\
Statutory sector employees & 38 & 75 \\
Basic grade & 31 & 61 \\
$>10$ years experience & 50 & - \\
\hline
\end{tabular}

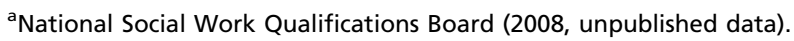

participants. A profile of the sample relative to the national workforce is presented in Table 1.

\section{Ethical approval}

As a multi-site study, ethical approval/exemption was provided by four committees including UCD Human Research Ethics Committee (Humanities).

\section{Data collection}

Phase I Audio-diaries: Building on the ABA-inspired approach, audiodiaries were used to capture the relatively unpredictable IB events within the constraints of a confidential environment. As indicated by post-study evaluation, this method accommodated a preference for orality, was more effective and less arduous for participants than written data collection and by capturing close to real-time data, reduced reliance on participant recall. For ten working days, diarists made narrative audio-recordings on an event-based schedule, describing all instances of social work practicerelated information behaviour. This study did not seek to gather client- or case-specific information (e.g. medical history), but rather sought to focus on information that would be carried forward to support future practice (e.g. information about a medical condition). Despite the limitations of relying on self-reported data from busy professionals, diarists returned diaries which were substantial, averaging 3,500 words. Recordings were transcribed in full, emerging questions noted in the margins and annotated transcripts forwarded to diarists. Acknowledging the limitations of selfreported diary methods, diaries were supplemented by CIT interviews.

Phase II Interviews: Building on Flanagan's (1954) CIT, the post-diary interviews used diary transcripts as real-world accounts to ground discussion of information behaviour in real-world events. These CIT interviews provided significant augmentation, yielding an increase in wordcount of 72 per cent on average. 


\section{Data analysis}

Thematic analysis was used to identify and elucidate issues. Subsequent coding of diaries, informed by IB models, allowed qualification of the various strategies used by diarists.

\section{Findings}

At an early stage of this study, it became clear that social workers may not always engage in the type of seeking described in IB models. Rather, while classic IB is used for some social work purposes, much of practitioners' IB is more pragmatic, truncated, fast-paced, shared and functional. Thus, existing information-seeking models served only to highlight the luxury that classic information seeking represents within social work practice and the need to consider a 'palette' of informationseeking approaches.

In order to capture the nature of social work IB a 'Model of Pragmatic Information Behaviour' (Figure 1) was devised. Building on Wilson's (1997) process model and drawing on discourses on the four dimensions, the model aimed to frame a holistic overview of professionals' IB using a 'palette approach' to each dimension. Within each dimension of the model a range of strategies, derived from theoretical models, reflect the varied nature of IB more typical of frontline workers. For example, the seeking strategies that social workers engage in such as monitoring, browsing or actively seeking are all included under the 'seeking' dimension of the model. In the interests of clarity, the various theories which informed the model are detailed alongside associated findings (below).

Using the 'Model of Pragmatic Information Behaviour', the findings from this study establish the information needs, seeking strategies, channels of acquisition and uses of participating diarists.

In all, the diarists recorded 424 information events (range 11-110) throughout the ten-day fieldwork period. These events ranged from the simple identification of an information need; through to actively seeking a piece of information; or applying a piece of acquired information to practice. Where more than one event was required to reach a resolution, these were grouped into sequences, yielding 253 information sequences.

\section{Function of social work IB}

The function of information in social work practice is broad ranging. Open-coding of the 253 IB sequences reported by diarists yielded five broad functions of information (Table 2). 


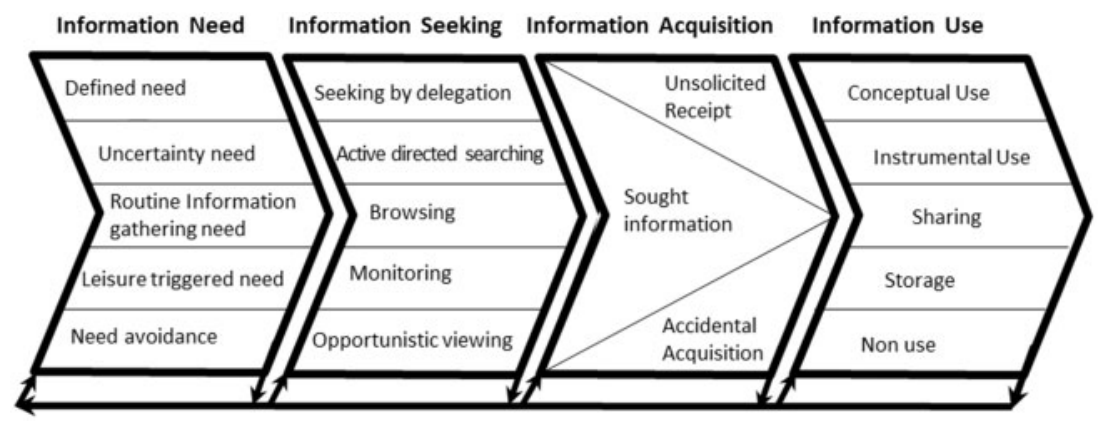

Figure 1: Model of Pragmatic Information Behaviour.

Table 2 Function of information sequences

\begin{tabular}{|c|c|c|c|c|}
\hline \multirow[t]{2}{*}{ Information sequence function } & \multicolumn{2}{|c|}{$\begin{array}{l}\text { Information } \\
\text { sequences }(N=253)\end{array}$} & \multicolumn{2}{|c|}{$\begin{array}{l}\text { Diarists engaging with } \\
\text { each sequence type }(N=16)\end{array}$} \\
\hline & $n$ & $\%$ & $n$ & $\%$ \\
\hline Case-directed & 109 & 43 & 16 & 100 \\
\hline Self-education & 48 & 19 & 13 & 81 \\
\hline Unsought information & 42 & 17 & 11 & 69 \\
\hline Professional information sharing & 27 & 11 & 10 & 63 \\
\hline Agency-related work & 27 & 11 & 8 & 50 \\
\hline
\end{tabular}

'Case-directed sequences', where information seeking was in response to a specific case, represented the most substantial function of IB and accounted for nearly half of information sequences.

'Self-education sequences', where seeking was aimed at keeping up-todate, and developing professionally, accounted for a fifth of IB.

'Unsought information sequences' refer to instances where information was received without any seeking on the part of the diarist, for example information was shared by a colleague. Over two-thirds of diarists received information in this way and it accounted for a sixth of information sequences.

'Professional information-sharing sequences', where information seeking was prompted by a request for information from another professional, was similarly prevalent with over two-thirds of diarists responding to information requests from colleagues. It is notable that information seeking to inform other professionals, combined with unsought information, accounted for over a quarter of information searching, indicating a high level of intra-professional information sharing.

'Agency-related work sequences', where the function of information seeking was to support administrative/policy work, was part of the IB of half of the diarists and accounted for a tenth of information sequences. 


\title{
Dimension 1: information needs
}

Thematic coding of diaries, informed by theoretical models, revealed five types of needs that prompted social workers to seek information (Figure 2). Two of these dominated.

'Defined-information needs' (Atkin, 1973), i.e. a need for a welldefined or known-item of information, accounted for a third of sequences. For example, one diarist reported:

[I rang] a support agency .... I was enquiring in relation to one of their parenting courses, about the starting date and some more details on the programme. The information was sought for a client who ... could benefit from some parenting support.

Alternatively, a defined search can be more complex, involving repeated iterations and multiple sources, while nonetheless involving searching for a known-item from known sources, e.g. seeking a service place for a client with challenging needs.

'Uncertainty information needs' (Dervin, 1983), i.e. where the social worker needs a greater understanding of something, rather than a specific item of information, accounted for a quarter of all sequences, for example

\begin{abstract}
... the family were at increased risk of foetal anomaly and it has triggered me to ... think about looking up to see if there is any relevant literature available on working with couples where fertility is an issue and where there have been previous foetal anomalies.
\end{abstract}

'Routine information gathering' (Wilson, 1977) such as monitoring journals was notably less evident (10 per cent). This, viewed in conjunction with the two preceding needs, indicated a clear dominance of reactionary needs, arising from ongoing work as opposed to planned or routine information gathering such as keeping up-to-date.

'Leisure-triggered information needs' (Godbold, 2006), e.g. looking for information following media coverage of an issue, while not common (6 per cent) are nonetheless evident.

'Information-need avoidance' (Sweeny et al., 2010) revealed an interesting conundrum: On the one hand, diary evidence of information needs which social workers failed to pursue was negligible (1 per cent). However, in CIT interviews, diarists almost unanimously indicated that they regularly experience information needs which they do not follow up. These needs appear to relate largely to issues of understanding, issues which are perhaps deemed less important by dint of not being required to justify actions. Perhaps the cost of acquiring this information is outweighed by its benefit? These findings seem to suggest the existence of a sub-strata of information gaps that are not being articulated as information needs. This may therefore represent information avoidance. 


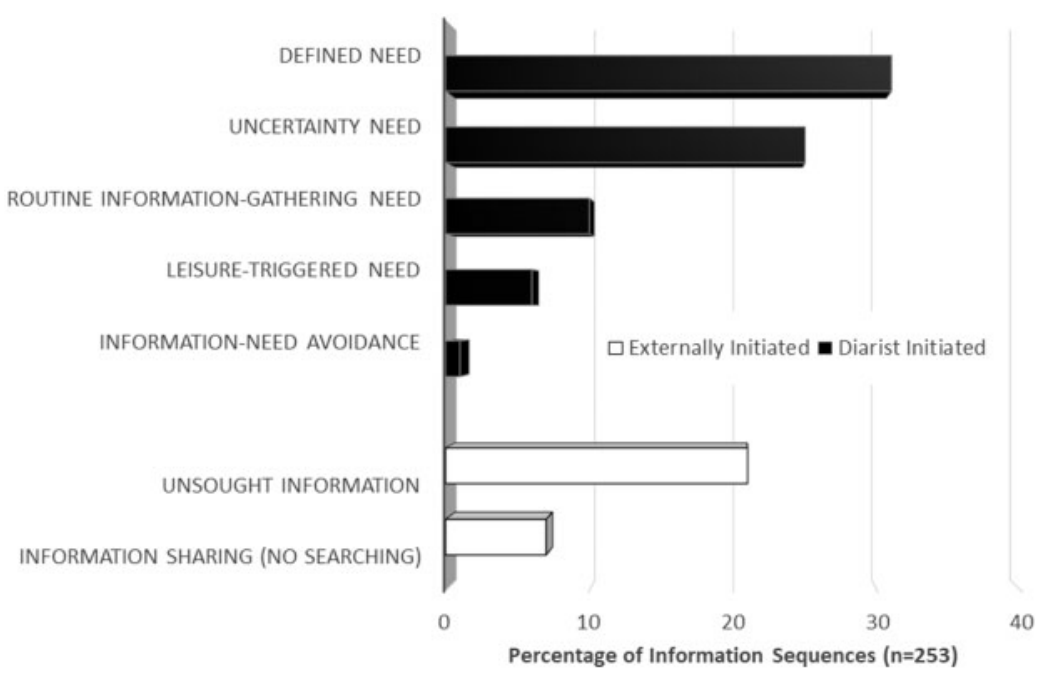

Figure 2: Nature of information needs.

'Externally initiated information sequences', where information was requested by another professional or was unsolicited and thus did not begin with an information need, accounted for over a quarter of sequences, again pointing to the prominent role of information sharing within the profession.

\section{Dimension 2: information-seeking behaviour}

Social workers' seeking strategies are addressed in the second dimension of IB (Figure 3).

'Delegation' (Niedzwiedzka, 2003), i.e. asking someone for information, emerged as the foremost search strategy, accounting for half of all searches, for example

[A scheme is relevant] to a patient that I have ... [and] I had to go to [my principal] and say ... "I'm going to need direction. I'm going to come back to you once I've done these bits, to check it".... [She emailed me] basic information on the 'how-to', step-by-step. ... We completed [the process] and that lady [availed of the scheme].

'Active-directed searching' (Bates, 2002), which involved accessing impersonal sources to pursue a specific goal, was less prevalent, accounting for 37 per cent of searching, for example

A child is missing from care ... so I have accessed the 'Children Missing in Care Report' ... from the HSE website - to look at the protocol. We've been going through that and fulfilling all of the tasks asked of us. 


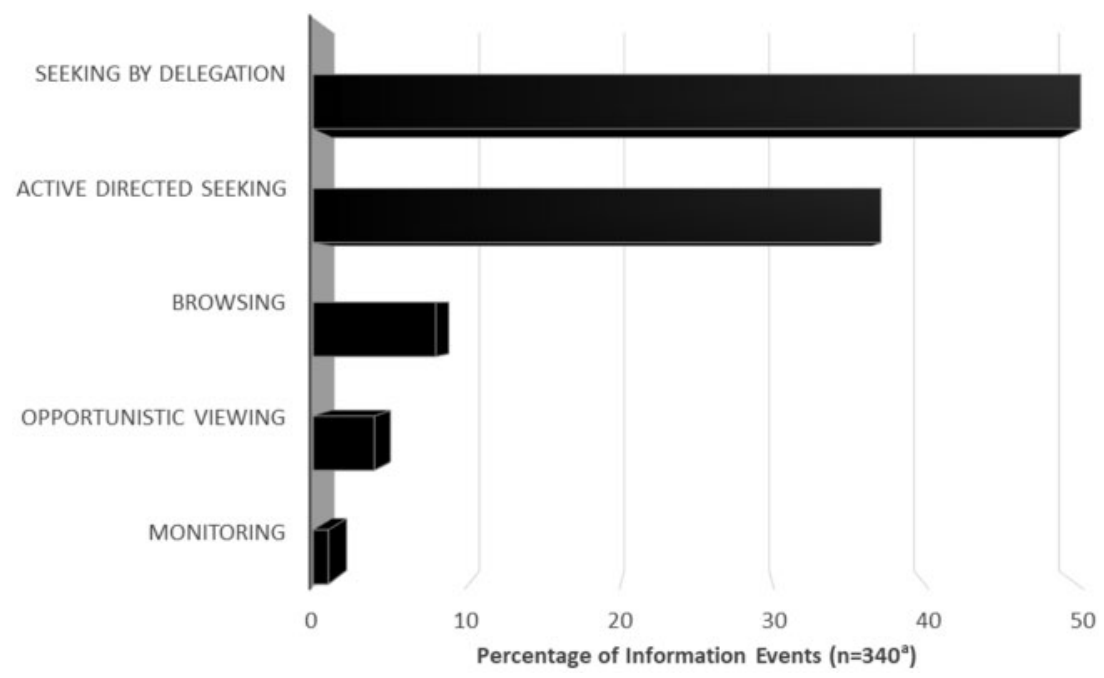

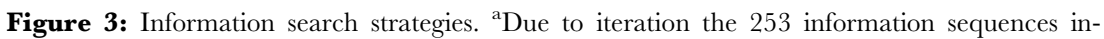
volved 340 events.

Less-directed approaches represented minority strategies in the diarists' toolkit. These included browsing-where the information need is fuzzy and less goal directed; viewing -i.e. 'happening on' information by accident; and monitoring-i.e. regular review of key sources.

Various aspects of stopping behaviour are illuminating. First, a sizeable majority ( $n=172,68$ per cent) of diarists' searching achieved resolution. Secondly, consideration of partly and unresolved issues revealed an almost exclusive intention not to stop searching until a resolution had been reached.

\section{Dimension 3: acquisition of information}

The third dimension of IB involves acquisition of information, encompassing unsolicited information, accidental acquisition (Erdelez, 1997) and the information seeking discussed earlier (Figure 4).

'Seeking information' was the predominant mode of information acquisition accounting for 82 per cent of fruitful information events. While negligible amounts of 'accidental acquisition' of information were identified, 'unsolicited receipt of information, e.g. sharing of information by others, accounted for 17 per cent $(n=62)$ of resolved information acquisition events. It is noteworthy that in-house colleagues were the most frequent source of unsolicited information ( $n=30,48$ per cent). 


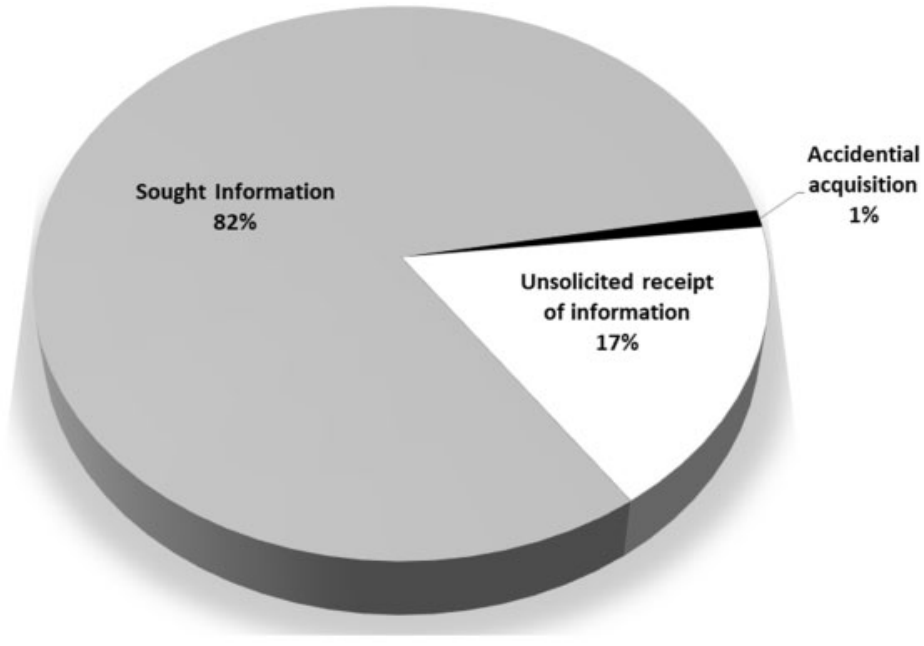

Figure 4: Mode of information acquisition (includes $\mathcal{N}=367$ resolved events).

\section{Dimension 4: information use}

The final dimension addresses use of acquired information to support practice. Five types of information use were identified (Figure 5). The various categories of use were not, however, mutually exclusive and in many instances the diarists put information to multiple uses, e.g. much of the information which was applied directly to practice was also shared with others. Taking a process approach, based on proximity to practice, it is possible to map the flow of information use (Figure 5).

'Instrumental application' (Kari, 2010), i.e. acting on acquired information or using the information to inform decision making, was observed in half ( $n=125,49$ per cent) of the information sequences. Moreover, the rate of application was even higher when the purpose of the IB was case-management $(n=45,68$ per cent) or when information was sought for a client $(n=35,81$ per cent).

If information was not applied to the seekers own practice it might be 'shared' with others. Twenty-two per cent of information was not applied but was shared. Viewed in conjunction with information which was applied and shared and with sequences involving unsought information, nearly two-in-every-three ( $n=151,60$ per cent) information sequences resulted in or from sharing of information.

If information was neither applied nor shared it might be 'taken on board conceptually', i.e. 'taking a mental note' (Godbold, 2006, p. 7). This was evidenced by 'verbing' (Dervin, 1993) such as reading, discussing or reviewing the information. Twenty per cent of information was 


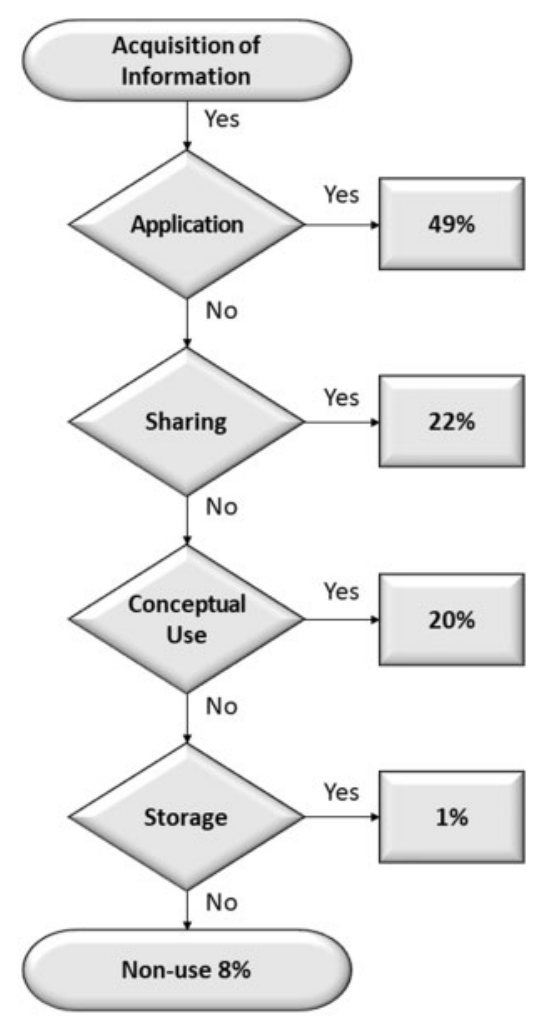

Figure 5: Flow-chart of information use.

retained conceptually for future use if it had no immediate application during this study's fieldwork.

If information was not employed in any of the above three ways, it might have been 'stored' unread. Only 1 per cent of information was stored without application, sharing or conceptual use, for example

[I received an] e-mail from a colleague on my team [about] ... The Wheel of Life it would be useful for [work] with clients. It's got things like decision making, problem solving, leadership skills. Looks good. That's all. Nothing further has been done with this piece of information but I added it to my favourites.

The phenomenon of acquiring more information than is required in the immediate-term is a feature of the information age. Moreover, with nearly one-in-five information sequences yielding unsolicited information, much of which cannot be applied immediately, the issue of storage of information has become important. It is likely that the diarists were relatively silent about storage of information. Once an item of 
information was used in some capacity, diarists may not have consistently recorded physical storage. The dominance of oral channels in this study may go some way to explaining the apparently low levels of physical storage.

Finally, if information was neither applied, shared, taken on board nor stored it is classified as 'non-use of information'. Only 8 per cent $(n=20)$ of sequences ended in non-use of information, mostly because the issue was unresolved at the end of the fieldwork. In only two instances a decision was made not to take onboard the information, for example

[Diary:] The general content of this e-mail was to say that a new issue was out. It's an online journal on all kinds of benefits and entitlements. ... [I] didn't look at the journal.

[Critical Incident Interview:] I never got back to this. [what stopped you?] Time. And I deleted it.

The fact that such a small proportion of acquired information went unused is indicative of highly efficient information practices among social work diarists.

\section{Diversity across dimensions}

Analysis across the four dimensions of IB revealed that diarists employed different strategies to address the two broad categories of casework and self-education. Case-related information searches involved a high level of specific, known-item searches, drawing on familiar sources, successfully resolved and applied. The reliance on familiar, synchronous, human sources before more formal sources, hints at a greater need for rapid responses and convenient, bite-sized information. This has the knock-on effect of placing an onus on colleagues, particularly supervisors, to be well-informed and supported with quality information resources.

Self-education searches were quite different. First, self-education was not as widespread, one-in-five diarists did not engage in self-education searches. In addition, self-education searches were more likely to be resolved in a single-event action, suggesting few barriers, but also little follow-up. It was interesting to see that diarists were as likely to actively search for self-education information as they are to acquire it by accident. Routine information gathering, including use of formal literature, was a minority search strategy. However, while many diarists did not engage in routine information gathering, it was a feature of other diaries, suggesting distinct personal differences. The approach to self-education 
would therefore appear to be somewhat ad hoc with such searches drawing on a significantly broader information base than casework searches.

\title{
Iteration in information behaviours
}

Many of the information events $(n=156,62$ per cent $)$ which diarists reported were stand-alone events encompassing trigger, need, seeking, acquiring and resolving (or abandoning) the information need, all in one event. In contrast, over a third of events $(n=97,38$ per cent) required a succession of events before resolution was achieved. For example, the following summary of a search sequence, which spanned a number of events over as many days, exemplifies how resolving the initial issue necessitated addressing a number of ancillary information needs and searches.

\begin{abstract}
A request from a former client for file-based information to support a court appearance involved the following: Identification of the staff member previously involved with the case; Location and review of the "closed file'; Discussion of the case with staff; Clarification of protocols for release of information; Requesting and confirmation of contact details for relevant medical and legal supports; Requesting signed consent from the client; Requesting an official 'request for release of information' from legal personnel; Releasing the information. [Summarised from original diary transcript]
\end{abstract}

The nature and causes of iteration highlight some of the challenges associated with social work information seeking. Few searches $(n=30,14$ per cent) were repeated because no relevant information was acquired in the initial search. A more common experience was to acquire information that contributed to the search, but require more information to complete the task ( $n=183,86$ per cent), for example

[I] had a prearranged [first] meeting with a client today in relation to guardianship, and access.... [T] he client had a lot of questions around managing if the situation becomes difficult between herself and the father of her baby. ... questions around a diagnosis of bi-polar depression. ... There was some discussion ... around how to work out an appropriate maintenance payment.

Such iterative information searches represent time-consuming and often invisible aspects of social work. One diarist commented on her diary transcript:

It [was] affirming. ... For one minor matter I made six phone calls [and] I came off the six phone calls feeling like I had gained nothing, but still I had put the effort into it. 


\section{Discussion}

Literature concludes that levels of researching and work-related reading among social workers are low. These claims beg the question, 'where do social workers get information to support their practice?' Many consider that social work relies on tacit knowledge. However, this implies that social workers do not typically seek out information, but rather absorb it as part of experience. Data from this study clearly indicate that social workers engage in a number of practice-related information-seeking events daily, but not typically research based.

There was considerable variation in the volume and patterns of diarists' IB: some engaged in substantial IB-e.g. seeking, monitoring, sharing and storing information-while others did not. This hints at an information-savvy cohort of social workers, akin to Allen and Cohen's (1969) 'Information Stars, who act as conduits to information for their colleagues. This personality type has long been recognised in literature (Pálsdóttir, 2010) and represents a key asset to the profession.

The corollary of identifying 'information stars' is that some practitioners may engage in little information seeking. While the small-scale and voluntary nature of participation in the diary-study make it difficult to draw robust generalisations about the amount of seeking, there would not appear to be evidence of a culture of seeking, or a culture of not seeking, but rather individual differences.

The principal information need of social workers is to inform a case. Self-education also features, although less prominently. A key finding of this study suggests that social workers' response to casework and selfeducation information needs are notably different from each other, requiring different strategies and practices. Moreover, while most social workers engage in case-related information seeking, not all engage in self-education. Perhaps efforts to increase information usage would be more effective if distinct strategies were applied to address these two disparate needs? Information for casework is needed fast and 'now', therefore it must be convenient and bite-sized, supported by data management and routine information gathering. In contrast, information for self-education would benefit from being manageable given limited time, but addressed by a more formalised, structured approach, in addition to being encouraged and promoted. Advances in continuous professional development and professional registration will undoubtedly contribute to a formalised approach to self-education information seeking.

Considering the triggers for information seeking, the predominance of needs for a defined piece of information, over needs for understanding or education, emerged. This type of reactionary information need is typically functional, facilitating progression of a case rather than drawing on 
effectiveness, evidence or reflection. This sits somewhat uneasily with the drive for evidence-informed practice.

The prevalence of delegation as an information-'seeking' strategy is clear. Interpersonal communication is, however, more dominant in caserelated searches than in other searches. This preference among social workers for interpersonal channels of information is well-documented in literature and it has been posited that the advantage of seeking by delegation is the flexibility and responsiveness of the source (Wilson, 1977). The appeal of delegation as a case-related information-seeking strategy may go beyond the issues of responsiveness and adaptability of interpersonal communication, to address issues of speed, convenience and confidence in the source, attributes which may not be as crucial to other searches. Moreover, the fact that Google searches which by their nature are quick, were also more prevalent in case-related searching, supports the idea that rapid response is important. The truncated nature of casework-related searching evident in this study, characterised by predominantly one-step searches, again underlines the emphasis on quick searches that rely on familiar sources.

In contrast to case-related searching, accessing impersonal sources (e.g. the Internet or books) to pursue a particular goal is most evident where the search aim was to inform other professionals or clients. Perhaps social workers go directly to the impersonal source when they were informing colleagues, but are more likely to ask for the information when informing themselves about case-related issues.

Delegation seeking strategies and a limited reading culture place an onus on colleagues, particularly supervisors, to be well-informed and supported with quality information resources. In this context, the potential of 'Information Stars' may be an important asset. Some general medical practice authors similarly suggest that pooling protected time in one post would be more productive (Young et al., 2006) while recognising that 'that those who are ... experts at teaching and providing clinical care are doing exactly what they should be doing' (Mainous and Hueston, 2006, p. 362).

Findings suggest that persistent pursuit and resolution of information searches may be a characteristic of social work IB. Evidence would seem to suggest that social workers do not stop pursuing information until they reach a resolution. In light of the issues of information-need avoidance and low readership, this begs the question 'do social workers only undertake searches that they consider resolvable within their existing resources?' Do searches that are considered irresolvable within existing resources remain in the sub-strata of gaps in knowledge which do not reach articulation as needs? Commensurate with the highly functional nature of social work searching, and the high-resolution rate, very little information sought by social workers remains unused or not stored. 
As such it would seem reasonable to conclude that social work information 'seeking' is highly efficient.

One of the recurrent features of social work IB is sharing or mutualeducation. However, the nature and frequency of sharing are not universal. Some departments have more formalised and active systems for information sharing than others. In addition to good storage practices to maximise the value of shared information, development and maintenance of quality shared resources may also be particularly effective in combating the risks of information satisficing, in essence a preventative strategy. The feasibility of info-share initiatives in terms of time, range of information and rate of information obsolescence varies from setting to setting and therefore requires setting-specific responses. The presence of a social work information star is, again, a clear advantage in this respect, as levels of sharing appear to be quite person-specific and not reflective of all social workers. However, systematic sharing of information remains one of the most innate and promising avenues for development of quality information.

Findings reiterate the conclusions of others that a reading culture is not widespread within social work (Buckley and Whelan, 2009; Iversen and Heggen, 2016). Encouragement and facilitation of reading of formal literature is required to develop a culture where reading is valued and undertaken. Initiatives ranging from drawing attention to available/open-access literature to sharing of learning can be undertaken at departmental level, or at a wider level if initiatives such as communities of practice, virtual communities, online and self-directed learning are harnessed. Pre-qualifying training in research must incorporate research literacy to support this.

At a general level, availability of time emerged as a key concern for informing practice and research has reported that social workers are 'information poor' by dint of a lack of time to read (Gannon-Leary, 2006, p. 122). Social workers, in this and other studies, have reiterated that in practice, particularly crisis intervention, rapid action takes priority over information seeking. Notwithstanding this, the exhortations from evidence-informed practice literature to integrate evidence and research findings into practice have continued. The impact of time restrictions has clear implications for practitioner's ability to seek information to support their practice. Perhaps it is time to put the issue of time, and all its practical and cultural components, centre stage and build a new approach to social work practice information around the issue of time. This will require profession-wide debate on innovative ways of promoting and integrating reading and continuous professional development.

\section{Conclusions}

Taking a palette approach to the needs, seeking, acquisition and use of information by social workers reveals key aspects of practitioner's 
information practices, aspects upon which a profession-specific response to information can be developed. These include the efficiency of IB; the centrality of colleagues in supporting information needs; the asset of information stars; propensity to share information; and different strategies for different social work tasks. These assets, building on what works for social work, may hold the key to addressing the need for a stronger reading culture and greater use of research evidence to support practice, both of which will go some way to addressing the possible avoidance of uncertainty information needs. Although literature on social work leadership in research capacity building 'remains meagre' (McDermott and Bawden, 2017, p. 902), it is encouraging to see the emergence of examples of such initiatives. For example, the Irish statutory Child and Family Agency has adopted 'Research and Information Mentors' in response to concerns about low levels of research (Tusla Child and Family Agency, 2017).

Perhaps the key recommendation from this study is the need for a profession-wide policy response, at both pre- and post-qualifying levels, to practitioners' information practices. Awareness building, promotion and encouragement of best practice in IB can build on the existing sharing practices which are characteristic of social work. Indeed, growth in agile working and associated technology usage may represent a boon in this regard (Jeyasingham, 2019). In attempting to address the topic of social work IB in a holistic fashion this study has cast a wide net. However, it is only through understanding how social work information needs evolve, and how social workers address these needs, that any advances can be made in improving dissemination and use of the information which is vital to support practice. Many aspects of this topic require further exploration before concrete solutions to the issue can be put in place. This study aspires to being a snap-shot of social work IB in action that will contribute to the construction of an effective and efficient system of information, which will in turn contribute to effective practice with the populations that are served.

The production of knowledge itself is not enough to guarantee that even the best knowledge will have any utility in practice and that we now need to search for more effective ways of generating and implementing new knowledge. (Kelly, 2017)

\section{Limitations of the study}

This study does not address the tacit-knowledge or critically reflective aspects of the knowledge base of social work. The purposive sample of this study is by definition a subjective, judgement-based sample; this may have yielded findings that represent a higher level of information use, research use and interest in the topic than evident generally. 


\section{Acknowledgements}

The research project was supported by Dr Valerie O'Brien and Dr Nessa Winston, University College Dublin, and this article benefited from the commentary and advice of Prof Jim Campbell, UCD, Dr Brian Melaugh and Professor Maurice Devlin, Maynooth University.

\section{Funding}

The research project was funded by the Government of Ireland Department of Children and Youth Affairs.

Conflict of interest statement. None declared.

\section{References}

Allen, T. J. and Cohen, S. I. (1969) 'Information flow in research and development laboratories', Administrative Science Quarterly, 14(1), pp. 12-19.

Atkin, C. (1973) 'Instrumental utilities and information-seeking', in Clarke, P. (ed) New Models for Mass Communication Research, Beverly Hills, SAGE. pp 205-42.

Avby, G., Nilsen, P. and Ellström, P. E. (2017) 'Knowledge use and learning in everyday social work practice', Child \& Family Social Work, 22(S4), pp. 51-61.

Bates, M. J. (2002) 'Toward an integrated model of information seeking and searching', International Conference on Information Needs, Seeking and Use in Different Context, Lisbon, 11-13 September.

Beddoe, L. (2011) 'Investing in the future: Social workers talk about research', British Journal of Social Work, 41(3), pp. 557-75.

Booth, S. H., Booth, A. and Falzon, L. J. (2003) 'The need for information and research skills training to support evidence-based social care', Learning in Health and Social Care, 2(4), pp. 191-201.

Buckley, H. and Whelan, S. (2009) Putting Research Evidence to Work: Key Issues for Research Utilisation in Irish Children's Services, Dublin, CAAB.

Buckley, H., Tonmyr, L., Lewig, K. and Jack, S. (2014) 'Factors influencing the uptake of research evidence in child welfare: A synthesis of findings from Australia, Canada and Ireland', Child Abuse Review, 23(1), pp. 5-16.

Case, D. O. and Given, L. M. (2016) Looking for Information: A Survey of Research on Information Seeking, Needs and Behavior, Bringley, Emerald Group.

Dervin, B. (1983) 'An overview of sense-making research: Concepts, methods, and results to date', Paper presented at the meeting of the International Communication Association, Dallas, Texas, May, available online at: http://faculty.washington.edu/ wpratt/MEBI598/Methods/An\%20Overview\%20of\%20Sense-Making\%20Research \%201983a.htm (accessed October 29, 2019).

Dervin, B. (1993) 'Verbing communication: Mandate for disciplinary invention', Journal of Communication, 43(3), pp. 45-54.

Ellis, D. A. (1993) 'Modeling the information seeking patterns of academic researchers', The Library Quarterly, 63(4), pp. 469-86. 
Erdelez, S. (1997) 'Information encountering: a conceptual framework for accidental information discovery', in Vakkari, P., Savolainen, R. and Dervin, B. (eds), Information Seeking in Context, London, Taylor Graham. pp. 412-21.

Fisher, K. E. (2005) 'Information grounds', in Fisher, K. E., Erdelez, S. and McKechnie, L. E. F. (eds), Theories of Information Behavior, New Jersey, American Society for Information Science and Technology and Information, Today Inc. pp. 185-90.

Fixen, D. L., Naoom, S. F., Blase, K. A., Friedman, R. M. and Wallace, F. (2005) Implementation Research: A Synthesis of the Literature, Florida, University of South Florida.

Flanagan, J. C. (1954) 'The critical incident technique', Psychological Bulletin, 51(4), pp. $327-58$.

Fook, J., Collington, V., Ross, F., Ruch, G. and West, L. (2015) Researching Critical Reflection: Multidisciplinary Perspectives, London, Routledge.

Gannon-Leary, P. (2006) 'Glut of information, dearth of knowledge? A consideration of the information needs of practitioners identified during the FAME project', Library Review, 55(2), pp. 121-31.

Godbold, N. (2006) 'Beyond information seeking: Towards a general model of information behaviour', Information Research, 11(4), available online at: http://informa tionr.net/ir/11-4/paper269.html (accessed October 29, 2019).

Gray, M., Sharland, E., Heinsch, M. and Schubert, L. (2015) 'Connecting research to action: Perspectives on research utilisation', British Journal of Social Work, 45(7), pp. 1952-67.

Greenhalgh, T. and Wieringa, S. (2011) 'Is it time to drop the 'knowledge translation' metaphor?', Journal of the Royal Society of Medicine, 104(12), pp. 501-9.

Harrison, J., Hepworth, M. and de Chazal, P. (2004) 'NHS and social care interface: A study of social workers' library and information needs', Journal of Librarianship and Information Science, 36(1), pp. 27-36.

Heinsch, M. and Gray, M. (2016) 'Making research count: What Australian social work researchers think', Australian Social Work, 69(4), pp. 428-42.

Heinsch, M., Gray, M. and Sharland, E. (2016) 'Re-conceptualising the link between research and practice in social work: A literature review on knowledge utilization', International Journal of Social Welfare, 25(1), pp. 98-104.

Holden, G., Barker, K., Rosenberg, G. and Cohen, J. (2012) 'Information for clinical social work practice: A potential solution', Clinical Social Work Journal, 40(2), pp. 166-74.

Horder, W. (2006) 'Reading' in professional practice: How social work practice assessors access knowledge and information' British Journal of Social Work, 37(6), pp. 1079-94.

Ingwersen, P. and Järvelin, K. (2005) The Turn: Integration of Information Seeking and Retrieval in Context, Dordrecht, Netherlands, Springer.

Iversen, A. C. and Heggen, K. (2016) 'Child welfare workers use of knowledge in their daily work', European Journal of Social Work, 19(2), pp. 187-203.

Jeyasingham, D. (2019) 'Seeking solitude and distance from others: children's social workers' agile working practices and experiences beyond the office', British Journal of Social Work, 49(3), pp. 559-76.

Kari, J. (2010) 'Diversity in the conceptions of information use', Information Research, 15(3), available online at: http://informationr.net/ir/15-3/colis7/colis709. html (accessed October 29, 2019). 
Kearney, A. J. (2008) Understanding Applied Behavior Analysis: An Introduction to ABA for Parents, Teachers and Other Professionals, London, Jessica Kingsley.

Kelly, L. (2017) 'Reconceptualising professional knowledge: The changing role of knowledge and evidence in social work practice', Social Work Education, 36(3), pp. 245-56.

Kuhlthau, C. C. (1991) 'Inside the search process: Information seeking from the user's perspective', Journal of the American Society for Information Science, 42(5), pp. 361-71.

Mainous, A. G. and Hueston, W. J. (2006) 'Is family medicine ready to move toward having professional researchers', Family Medicine, 38(5), pp. 361-2.

McDermott, F. and Bawden, G. (2017) 'New ways of seeing: Health social work leadership and research capacity building', Social Work in Health Care, 56(10), pp. 897-913.

Niedzwiedzka, B. (2003) 'A proposed general model of information behaviour', Information Research, 9(1), Paper 164, available online at: http://informationr.net/ ir/9-1/paper164.html (accessed October 29, 2019).

Pálsdóttir, A. (2010) 'The connection between purposive information seeking and information encountering: A study of Icelanders' health and lifestyle information seeking', Journal of Documentation, 66(2), pp. 224-44.

Randall, J. (2002) 'The practice-research relationship: A case of ambivalent attachment?', Journal of Social Work, 2(1), pp. 105-22.

Rosen, A. (2003) 'Evidence-based social work practice: Challenges and promise', Social Work Research, 27(4), pp. 197-208.

Sharland, E. (2012) 'All together now? Building disciplinary and inter-disciplinary research capacity in social work and social care', British Journal of Social Work, 42(2), pp. 208-26.

Sweeny, K., Melnyk, D., Miller, W. and Shepperd, J. A. (2010) 'Information avoidance: Who, what, when and why', Review of General Psychology, 14(4), pp. 340-53.

Tozer, C. L. and Ray, S. (1999) '20 questions: The research needs of children and family social workers', Research, Policy and Planning, 17(1), available online at: http://ssrg.org.uk/rpp-17-1-carol-1-tozer-20-questions-the-research-needs-of-chil dren-and-family-social-workers/ (accessed October 29, 2019).

Trevithick, P. (2008) 'Revisiting the knowledge base of social work: A framework for practice', British Journal of Social Work, 38(6), pp. 1212-37.

Tsang, N. M. (2007) 'Orality and literacy: Their relevance to social work', Journal of Social Work, 7(1), pp. 51-70.

Tusla Child and Family Agency (2017) 'Research and Information Mentor Strategy' [Online], available online at: http://www.tusla.ie/uploads/content/National_ Research_Office_Research_and_Information_Mentor_Strategy_Document_ Aug2017.pdf (accessed October 29, 2019).

Walter, I., Nutley, S., Percy-Smith, J., McNeish, D. and Frost, S. (2004) Improving the Use of Research in Social Care, London, SCIE.

Wilson, P. (1977) Public Knowledge, Private Ignorance: Toward and Library and Information Policy, Westport, CT, Greenwood Press.

Wilson, T. D. (1997) 'Information behaviour: An interdisciplinary perspective', Information Processing \& Management, 33(4), pp. 551-72. 
Wilson, T. D. and Streatfield, D. (1977) 'Information needs in local authority social services departments: An interim report on project INISS', Journal of Documentation, 33(4), pp. 277-93. 10.1108/eb026646.

Young, R. A., DeHaven, M. J., Passmore, C. and Baumer, J. G. (2006) 'Research participation, protected time, and research output by family physicians in family medicine residencies', Family Medicine, 38(5), pp. 341-8. 